given and not guessing at strengths or quantities. The amount of zinc acetate recommended is a safe excess. Sodium acetate does not appear to work as satisfactorily.

For the assay of alloys, etc., the necessary modifications of the foregoing scheme are obvious.

The foregoing scheme directs the use of five cc. of dilute nitric acid for dissolving the copper previous to titration and prescribes six to seven grams, or about twenty cc. of a saturated solution of zinc acetate as a safe excess of neutralizing agent. It is obvious that if most of the nitric acid be boiled away the amount of zinc salt necessary is greatly reduced. In such a case, however, it is perhaps best, for safety's sake, not to use less than one-half the prescribed quantity. Half the zinc salt may thus be saved at the expense of a little more time. This is the ordinary practice in my own laboratory.

DENVER, COLORADO.

\title{
ON TWO SOURCES OF ERROR IN SUGAR HOUSE ANALYSES.
}

BY EDMUND C. SHOREY.

Received March ro, 1896 .

INCIDENTAL to the study of the action of water of different temperatures on bagasse, I have had occasion to make some determinations which throw some light on a source of error in fiber determinations.

Finely cut cane was washed with cold water until the polariscope reading in a twenty $\mathrm{cm}$. tube was 0 ; the excess of water squeezed out in a press, and three portions of fifty grams each taken. In one the moisture was determined by drying to constant weight in an air bath at $105^{\circ} \mathrm{C}$.; the two others were treated with boiling water; one for five minutes and the other for two hours. The quantity of water used was in each case just sufficient to cover the cane chips, and at the expiration of the time the water and the chips were poured on a filter and washed four times with cold water, using each time the same quantity of wash water as was used in the original treatment. The filtrate so obtained was evaporated to dryness over a waterbath, dried at $105^{\circ} \mathrm{C}$, and calculated to percentage of dry sub- 
stance left after the exhaustion of sucrose with cold water. The filtrates obtained were decidedly milky, and this no amount of filtering would remove. A portion of filtrate on standing undisturbed for six days had not become less milky, and had thrown down no deposit.

The cane was prepared by cutting to fineness of saw dust in a meat chopper, and determinations have in all cases been made in duplicate.

The mean of six fairly mean concordant determinations is as follows :

(1) Treatment for five minutes with boiling water extracted seven and two-tenths per cent. of dry substance.

(2). Treatment for two hours with boiling water extracted twelve per cent. of dry substance.

To show the amount of error likely to result from using varying quantities of water and varying the time of boiling; suppose that the fiber and dry substance from the treatment with cold water was ten per cent. of cane, we would have:

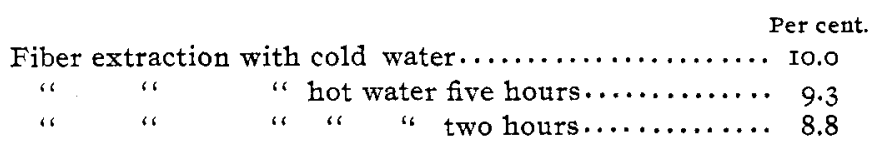

Dr. Beeson, of Audubon Park Sugar School, has pointed out in this Journal the errors in fiber determinations likely to result from the varying amounts of fiber in different parts of the same stalk of cane. This can be overcome, I think, by taking a sufficiently large sample and reducing it to a very fine state of division; but if fiber determinations by different chemists are to be comparable, some uniform method must be agreed upon. This method should provide for the fineness of division of cane or bagasse, the quantity of sample to be taken, the quantity of water to be used, the number of times washing, and the length of time of boiling.

In calculating the extraction by formulas similar to those given by M. Trubek in this Journal (Dec., I895), I prefer to dry the residue from alcoholic extraction and call this fiber. The object being simply to compare the fiber in the cane with that in the 
bagasse, and this method I find gives more uniform results with a minimum of labor.

A second source of error in sugar house analyses to which I wish to draw attention is in the determination of albuminoid nitrogen as commonly carried out.

In the official method for albuminoid nitrogen in finding stuffs the directions are to heat to boiling, add cupric hydrate, filter when cold and wash with cold water. In the determinations of albuminoid nitrogen in cane juice made by W. Maxwell, and published by him in a Bulletin of Louisiana Experiment Station, this method was modified, but for what reason I am unable to say. According to this modified method the juice was heated to boiling, cupric hydroxide added, boiled ten minutes, filtered hot and washed with hot water. Both these methods introduce a serious error, owing to the fact that when cane juice in its natural acid condition is heated a change is brought about in the aluminoid nitrogen contained therein, and in consequence the amount of aluminoids as determined by either of these methods is not really that which is in the juice in the plant, but that which is left from the action of heat and the acid in the juice.

During the past two years I have made a large number of determinations, as follows: I. Total nitrogen in juice; II. Albuminoid nitrogen in cold juice; III. Albuminoid nitrogen in juice which had been heated to $71^{\circ}-72^{\circ} \mathrm{C}$.; IV. Albuminoid nitrogen in juice which had been boiled one minute. For total nitrogen the Gunning method was used, and for albuminods cupric hydroxide was added to cold juice, filtered cold and washed with cold water, the precipitate and filter paper treated by Gunning method. In each case twenty cc. of juice have been used, and the results calculated to percentage of total solids as given by Brix spindle; the difference between total nitrogen and albuminoids has been called amides and calculated to asparagins.

As the results have been the same in all cases, I give below two analyses which I have already communicated to the Planter's Monthly, (Honolulu). 
I. Brix, 17.3; Purity, 9I.

Acidity, I00 cc. juice $=$ ten cc. $\frac{\mathrm{N}}{\mathrm{IO}}$ alkali.

Total nitrogen I. I I5 per cent. of total solids.

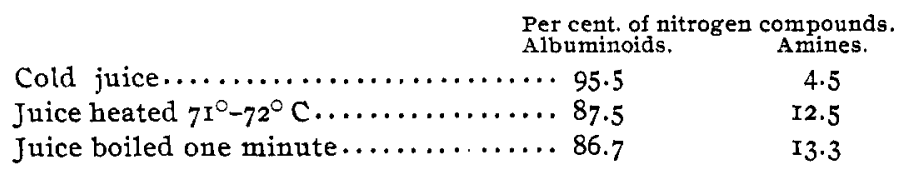

2. Brix, I8.2; Purity, 90.9.

Acidity, roo cc. juice $=$ I $.3 \mathrm{cc} . \frac{\mathrm{N}}{10}$ alkali.

Total nitrogen $\mathrm{I} .032$ per cent. of total solids.

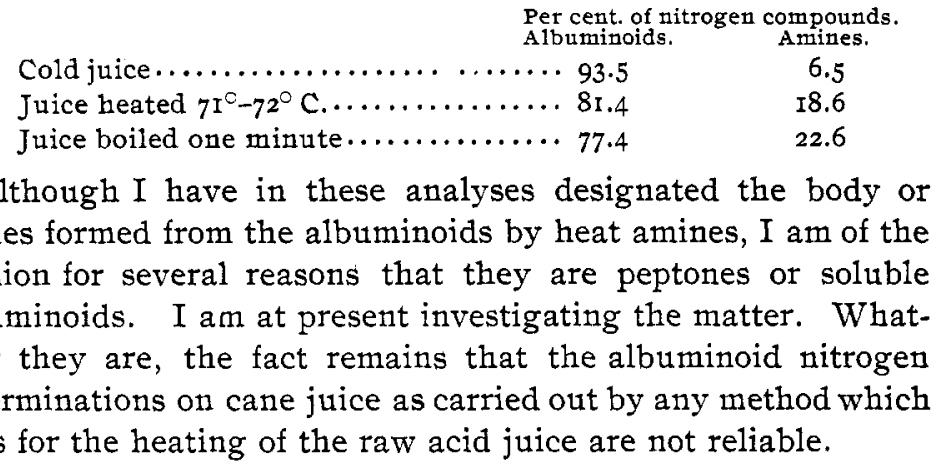

LABORATORY KOHALA SUgar Co.,

KOHALA. HAWAIT. 